\title{
Near infrared hyperspectral imaging system for root phenotyping
}

\section{Thomas Arnold, Raimund Leitner, Gernot Bodner}

Thomas Arnold, Raimund Leitner, Gernot Bodner, "Near infrared hyperspectral imaging system for root phenotyping ," Proc. SPIE 10217, Sensing for Agriculture and Food Quality and Safety IX, 102170D (1 May 2017); doi: 10.1117/12.2262441

EDent: SPIE Commercial + Scientific Sensing and Imaging, 2017, Anaheim, CA, United States 


\title{
Near Infrared Hyperspectral Imaging System for Root Phenotyping
}

\author{
Thomas Arnold ${ }^{\mathrm{a}}$, Raimund Leitner ${ }^{\mathrm{a}}$, and Gernot Bodner ${ }^{\mathrm{b}}$ \\ ${ }^{a}$ CTR Carinthian Tech Research AG, Europastrasse 12, 9524 Villach, Austria \\ bUniversity of Natural Resources and Life Sciences Vienna, Konrad Lorenz-Strasse 24, 3430 \\ Tulln, Austria
}

\begin{abstract}
This paper presents the development and application of a hyper-spectral imaging system for root phenotyping. For sustainable plant production root systems optimized for growing conditions in the field are required. Therefore, the presented system is used for the research in the field of plant drought resistance. The system is used to acquire spatially resolved near infrared (NIR) spectroscopy data of rhizoboxes. In contrast to using visible light $(380 \mathrm{~nm}-780 \mathrm{~nm})$ the NIR wavelength range $(900 \mathrm{~nm}-1700 \mathrm{~nm})$ allows to discriminate essential features for the root segmentation and water distribution mappings. The increased image contrast in the NIR range allows roots to be segmented from soil and additional information, e.g. basic root-architecture, to be extracted. In addition, the water absorption bands in the NIR wavelength range can be used to determine the water content and to estimate the age of the roots. In this paper the hardware setup of the hyper-spectral root imaging system, the data analysis, the soil water content estimations and the root segmentation using different methods to optimize separation between roots and soil, both constituting complex materials of variable properties, are presented.
\end{abstract}

Keywords: Root Phenotyping, Hyperspectral Imaging, Imaging Spectroscopy

\section{INTRODUCTION}

In modern agriculture drought is a major cause of low yields worldwide. There is an urgent need for more water efficient cropping systems facing large water consumption of irrigated agriculture and high unproductive losses via runoff and evaporation. ${ }^{1}$ Thus, a better understanding of the key functionality of roots can lead to a better management of agricultural ecosystems. The main objective of root measurement is to obtain new knowledge on key functioning of roots. The key challenges in root measurements are that often sampling is destructive, that roots show high spatio-temporal variability making sampling design difficult, the need for high throughput e.g. for screening genotypes in a breeding context. ${ }^{2}$ An other important aspect is that the roots should be measured in their natural soil environment to obtain information not only on root architecture but also on root functioning. Therefore, imaging systems that enable to study the interactions between plant and soil are a way towards better crop water supply. Especially the water distribution in very close proximity of the roots, the so called rhizosphere, is of central importance. Within this research project a hyperspectral root imaging system for the acquisition of hyper-spectral NIR image data of rhizoboxes (see Fig. 1) was developed. The system is used as an imaging modality for research activities in the field of plant drought resistance. In contrast to imaging using visible light, hyper-spectral NIR imaging allows us to achieve a higher contrast between roots and the soil for a more reliable segmentation of the roots against the soil. Moreover, it is possible to visualize the water distribution in the soil and the roots. And it is possible to acquire time-lapse images to monitor changes in water distribution or water uptake over time. With the current setup a wide variety of different soil and crop combinations can be investigated. This imaging modality can deliver important information for the development of drought resistant crops.

Further author information: (Send correspondence to Thomas Arnold: E-mail: thomas.arnold@ctr.at)

Sensing for Agriculture and Food Quality and Safety IX, edited by M. S. Kim, K. L. Chao, B. A. Chin, B.-K. Cho, Proc. of SPIE Vol. 10217, 102170D (c) The Authors. Published under a Creative Commons Attribution CC-BY 3.0 License · doi: 10.1117/12.2262441

Proc. of SPIE Vol. 10217 102170D-1 


\section{MATERIALS AND METHODS}

The conditions at the soil-root interface are sometimes considerably different from those at a distance from the roots, and many researchers have been interested in studying the characteristics of this zone (rhizosphere) in relation to bulk soil. ${ }^{3}$ To be able to study the soil-root interface it is necessary to cultivate the crops in rhizoboxes and measure the water distribution non-destructively.

Rhizobox system A rhizobox or root box system allows observation of root growth in a natural soil medium and over longer time spans. This enables to study different aspects of soil-root interactions like root growth, depth distribution and water distribution. Moreover, it is possible to sample different age classes or orders of roots. A rhizobox consists of a rigid frame and contains a layer of soil with a certain depth. The front face of the rhizobox consists of a transparent window. The backside is non-transparent and usually contains irrigation channels. During growth of the crops the glass window is covered and the rhizobox is mounted at an angle to ensure that the roots grow towards the glass window. The glass window makes it possible to study the inside of the rhizobox without the risk of dehydration of the roots and the soil during measurement.
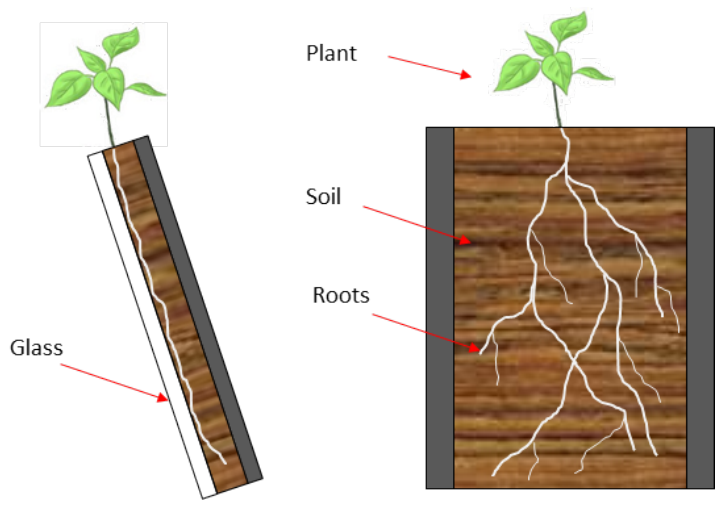

Figure 1. (left) Side view of a rhizobox, (right) Front view of a rhizobox

The rhizoboxes used for this work had a length of $1000 \mathrm{~mm}$ a width of $300 \mathrm{~mm}$ and a depth of $10 \mathrm{~mm}$ to $30 \mathrm{~mm}$. For the different experiments a variety of soils was evaluated. For example field top soil (silt loam) was sieved to a particle size of smaller than $2 \mathrm{~mm}$ and filled horizontally to a bulk density of $1.3 \mathrm{~g} / \mathrm{cm}^{-3}$. After filling the rhizoboxes were planted with one seed of durum wheat that was pre-germinated for 3 days at $20^{\circ} \mathrm{C}$. The plants were grown in a growing chamber equipped with LED illumination. Daylight was set to 14 hours and day-night temperature was $23^{\circ} \mathrm{C}$ and $16^{\circ} \mathrm{C}$ respectively. The rhizoboxes were stored at an $45^{\circ}$ angle. After approximately 60 days the roots of the plants reached the bottom of the rhizobox.

Hyperspectral Imaging (HSI) is the combination of machine vision with spectroscopy and comprises the acquisition, processing and classification of hyper-spectral images. ${ }^{4}$ Compared to an RGB camera that captures three broadband channels, a spectral imaging system captures up to several hundred narrow band channels. ${ }^{5}$ The increased spectral information makes chemometric analyses possible. For this application a spatial scanning SI approach was chosen. Spatial scanning HSI is more suitable for many high throughput applications. Instead of recording a two-dimensional image like a wavelength-scanning HSI, a line across the sample, perpendicular to the direction of the relative movement, is projected into an imaging spectrograph. The spectral information for each pixel along this observation line is projected along the second axis of the two-dimensional detector chip. The spectral encoding can be provided by an imaging spectrograph (see Fig. 2). Since the spatial information along the line is retained, the images contain the spatial information along the first axis and the full spectral wavelength information along the second axis. ${ }^{6}$ The spectral and the first spatial dimension are simultaneously acquired, while the second spatial dimension is recorded sequentially due to the movement of the sample relative to the HSI sensor. The HSI data acquisition process produces a three-dimensional dataset with two spatial 
dimensions and one spectral dimension. For each image pixel a NIR reflectance spectrum is available. Due to the high spatial resolution of the scan and the limited size of the NIR camera detector the whole rhizobox has to be scanned in several strides as shown in Fig. 6 .

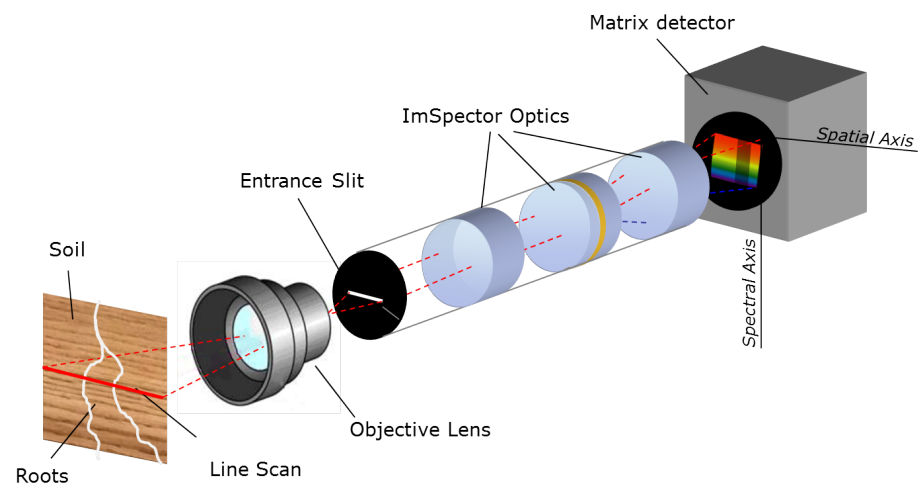

Figure 2. Imspector $^{\circledR}$ (Specim, FI) imaging spectrograph

The individual strides have then to be combined to a registered dataset. For a rhizobox with the dimensions of $1000 \mathrm{~mm}$ by $300 \mathrm{~mm}, 10$ strides have to be acquired. Each stride has a pixel size of 320 pixel by 10000 pixel.

\section{MEASUREMENT SETUP}

The hyperspectral root imaging system consists of a hyperspectral imaging sensor (see Fig. 3) and a 2D scan system. The hyperspectral imaging sensor consists of (i) a cooled 14-bit monochrome NIR camera (Xenics, BE) with a spectral range from $900 \mathrm{~nm}$ to $1700 \mathrm{~nm}$, a pixel resolution of 320 pixel by 256 pixel and a frame rate of $100 \mathrm{~Hz}$, (ii) an Imspector ${ }^{\circledR}$ N25E imaging spectrograph (Specim, FI) with a spectral range from $900 \mathrm{~nm}$ to $2500 \mathrm{~nm}$ and a spectral resolution of $5 \mathrm{~nm}$ (iii) and a halogen line illumination that is arranged in a $+45^{\circ} /-45^{\circ}$ geometry.

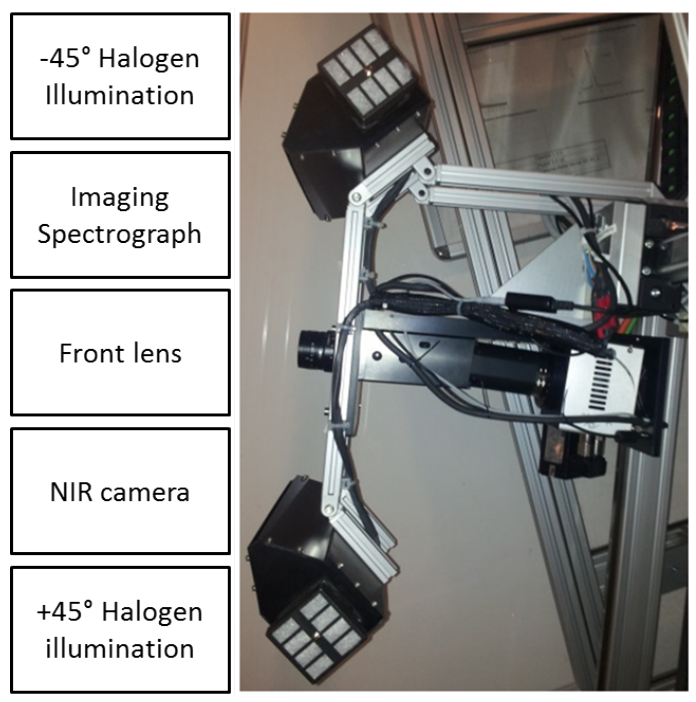

Figure 3. Hyperspectral imaging sensor

To scan the whole area of a rhizobox the imaging sensor has to be moved. This is done by the 2D scan system shown in Fig. 4. The two axes of the scan system are tilted by an angle of $30^{\circ}$ to allow long term measurements. The rhizobox mount is adjustable to allow for measurements of rhizoboxes with different dimensions. For the 
calibration of the imaging sensor a white standard is mounted above the rhizobox. This white standard in combination with a dark standard is necessary to calibrate the acquired image data for inhomogeneities of the light source and defective pixels and electronic noise of the detector.

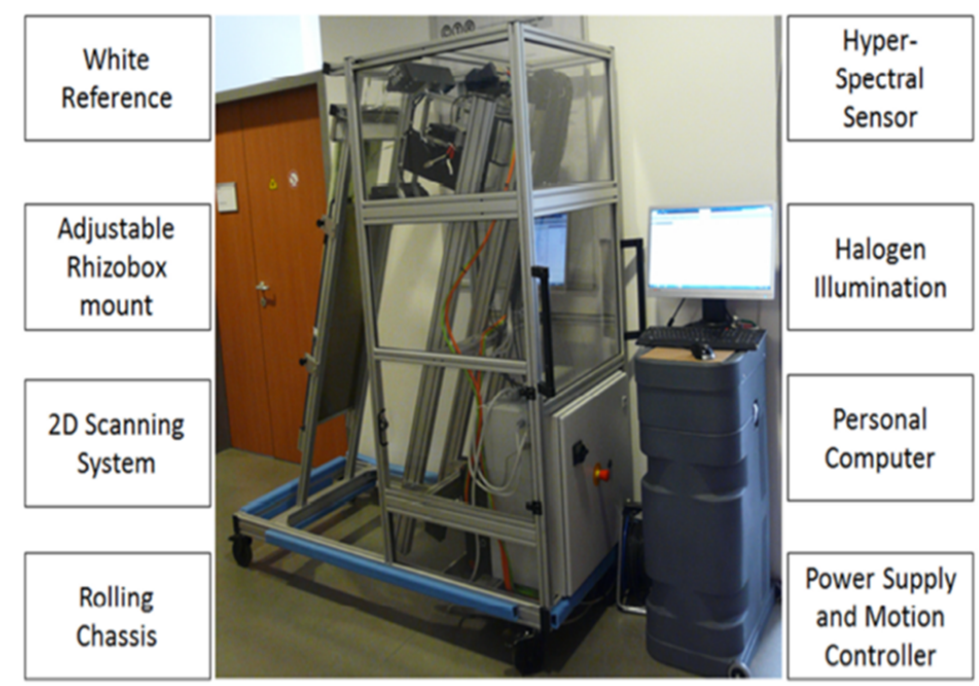

Figure 4. Measurement setup: hyperspectral root imaging system

\section{RESULTS}

The hyperspectral NIR root imaging system was evaluated with a variety of different soil substrates, ranging from fine sand to coarse, dark soils. The acquired hyperspectral datasets contain data with a spectral range from $900 \mathrm{~nm}$ to $1700 \mathrm{~nm}$ projected across 256 pixels. For evaluation, the spectral information is hence separated into 256 narrow-band images. In Fig. 5 normalized NIR reflectance spectra of different areas inside a rhizobox are shown.

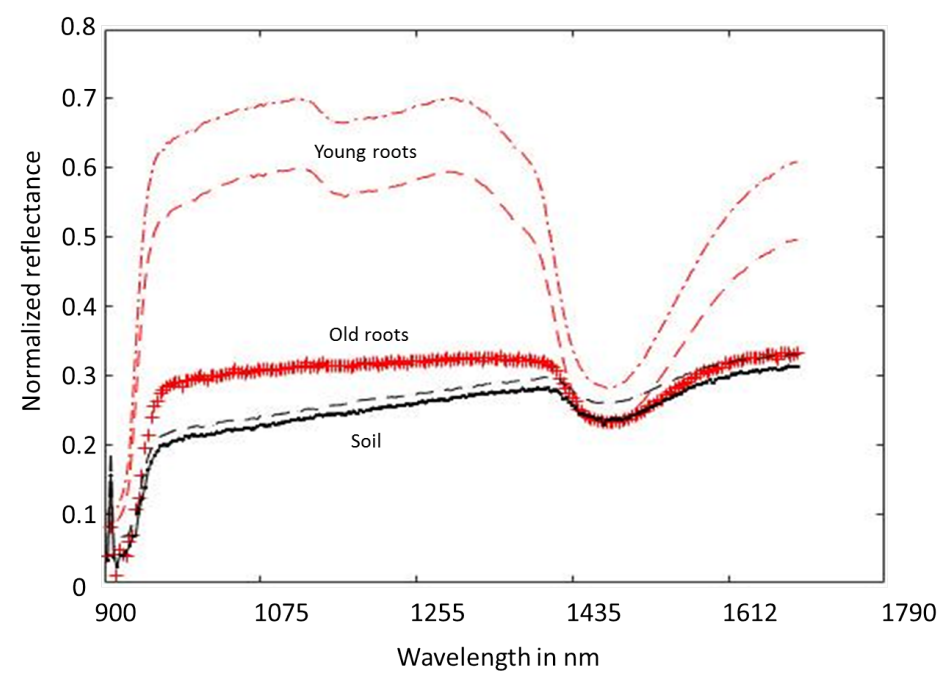

Figure 5. NIR reflectance spectra of different areas inside a rhizobox

It is visible that young roots have a higher reflectance over the whole wavelength range than old roots and soil. At approx. $1450 \mathrm{~nm}$ a decrease in the spectral response is visible which is due to a water absorption band. 
Another water absorption band is visible in the spectra of the young roots in the spectral region from $1100 \mathrm{~nm}$ to $1300 \mathrm{~nm}$. The high spectral resolution makes it possible to isolate information that is related e.g. to the water content. Moreover, due to the increased contrast in the NIR image the segmentation of the roots from the soil can be done more reliably than in the visible wavelength range. Especially for difficult cases like old roots on dark soil or young roots from bright sand. In Fig. 6 a NIR image of young roots in sand is shown at a wavelength of $1450 \mathrm{~nm}$.
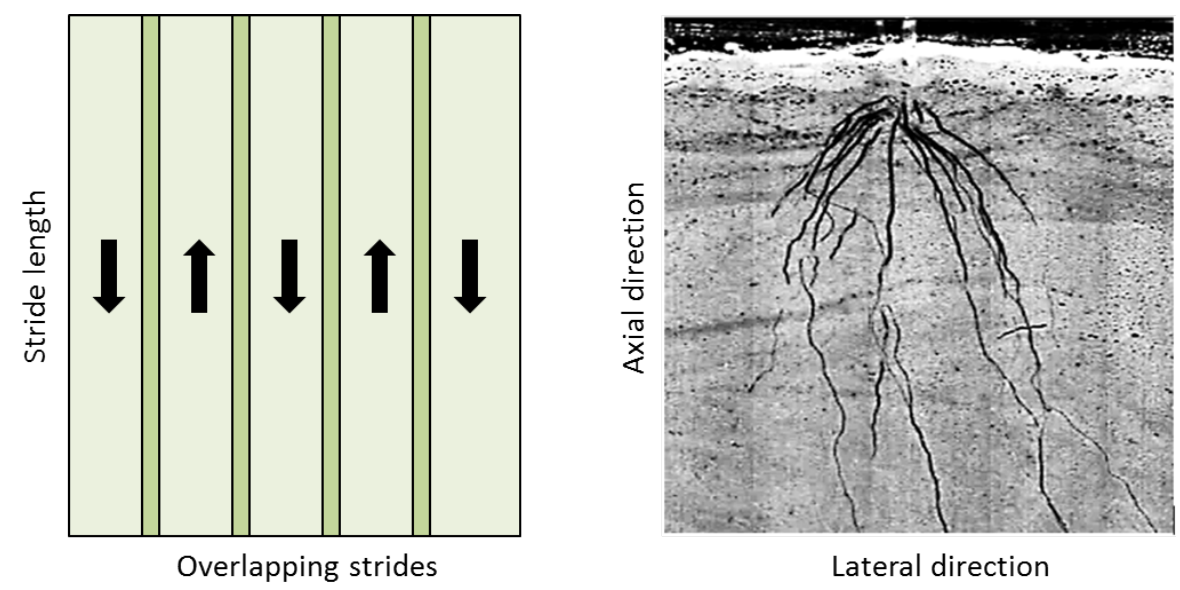

Figure 6. (left) The imaging system acquires image strides with a width of $30 \mathrm{~mm}$ and a horizontal overlap of $5 \mathrm{~mm}$. The strides are acquired in a meander scan pattern. (right) NIR image of roots in a rhizobox consisting of five image strides. The absorption at $1450 \mathrm{~nm}$ is shown as monochrome information.

Due to the enhanced contrast in the NIR image, the segmentation of the roots against the sand substrate is significantly improved. For water distribution measurements, a rhizobox with 8 soil layers was prepared. The soil layers were separated from each other and contained different amounts of water, ranging from 0.0 grams to 0.35 grams of water per gram of soil. Fig. 7 shows the two-dimensional distribution of the calculated water content.
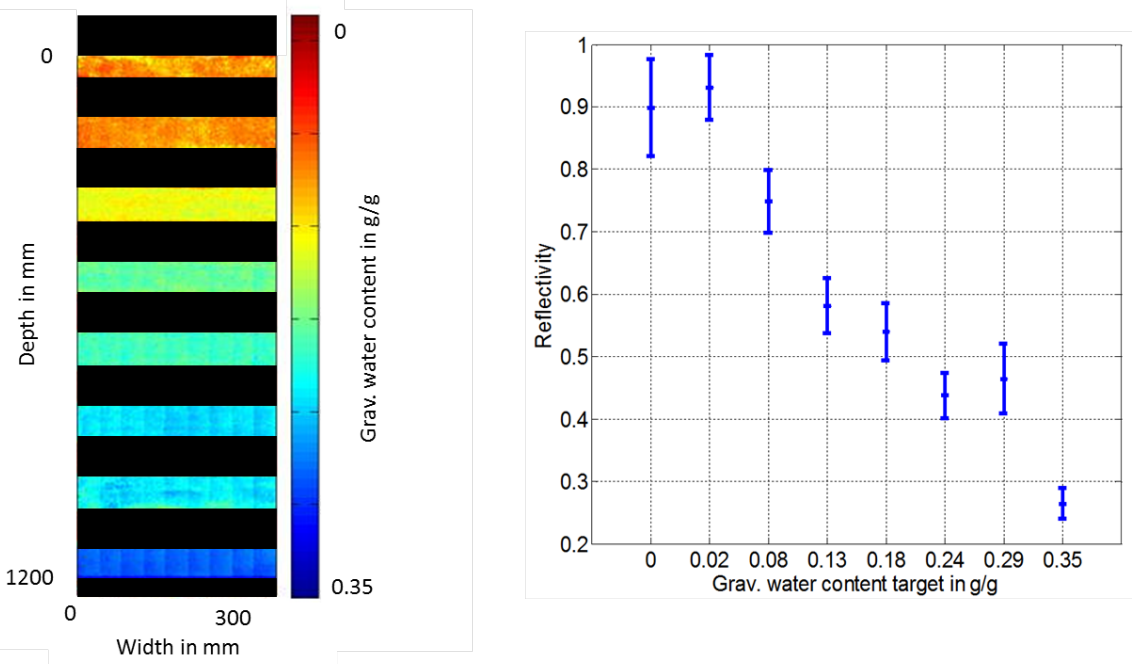

Figure 7. (left) Water content calibration measurements of a rhizobox comprising soil layers with different water contents, (right) Error-bar plot of the reflectivity at $1450 \mathrm{~nm}$ for regions of different water content in a rhizobox.

For the water content measurements it was necessary to derive the calibration curves for each substrate indi- 
vidually. The correlation between the measured signal intensity at $1450 \mathrm{~nm}$ and the gravimetric water content is shown in Fig. 7. The error-bar plot shows a linear correlation between the reflectivity data and the gravimetric water contend. However, it is visible that two of the error-bars are slightly offset. This can be due to inhomogeneities in the water distribution inside the corresponding layers of the rhizobox. This means that the gravimetric water content was correct for the soil layer in the rhizobox but on the surface that was measured it was higher. A possible reason for this effect can be water condensation on the inside of the glass. It was also observed that soils with larger particle size or inhomogeneous composition may also lead to less precise optical water content measurements.

\section{CONCLUSIONS \& OUTLOOK}

We presented a hyperspectral imaging system for the investigation of rhizoboxes containing roots and soil. The system was evaluated on a variety of different substrates and water contents. Initial results of the calibration measurements for the determination of the soil water content have been presented. The system has proven to be a valuable tool for the investigation of the water distribution within a rhizobox. The developed hyperspectral imaging system is now used at the University of Natural Resources and Life Sciences Vienna for the characterization of the root system architecture of agricultural crops. The goal is to find agricultural crops that are more efficient in their water uptake and also provide a high crop yield under environmental stresses such as drought. Future investigations will focus especially on the water distribution in very close proximity of the roots, the so called rhizosphere. We expect that the developed system will enable breeders to make a targeted choice of agricultural crop species with adequate root characteristics to improve their water uptake capacity and maximize their crop yield.

\section{ACKNOWLEDGMENT}

The development of the hyperspectral imaging system was conducted in part within the K1 competence center ASSIC (Austrian Smart Systems Integration Research Center), which is funded within the R\&D Program COMET - Competence Centers for Excellent Technologies by the Federal Ministries of Transport, Innovation and Technology (BMVIT), of Economics and Labour (BMWA) and it is managed on their behalf by the Austrian Research Promotion Agency (FFG). The Austrian provinces (Carinthia and Styria) provide additional funding. The hyperspectral rhizobox scanning system and the studies aiming at water distribution measurements inside a rhizobox were conducted as part of the FWF project "The roots of drought resistance (P 25190-B16)".

\section{REFERENCES}

[1] Bodner, G., Nakhforoosh, A., and Kaul, H., "Management of crop water under draught: areview," Agronomy for Sustainable Development 34 (04 2015).

[2] Smit, A., Bengough, A., Engels, C., van Noordwijk, M., Pellerin, S., and van de Geijn, S., [Root methods: a handbook], Springer Science \& Business Media (2013).

[3] Youssef, R. A. and Chino, M., "Development of a new rhizobox system to study the nutrient status in the rhizosphere," Soil Sci. Plant Nutr. 34(3), 461-465 (1988).

[4] Bearman, G. H., Nelson, M. P., and Cabib, D., "Spectral imaging: Instrumentation, applications, and analysis," Proc. SPIE International Society for Optical Engeneering (2000).

[5] Leitner, R., Biasio, M. D., and Arnold, T., "High-sensitivity hyperspectral imager for biomedical video diagnostic applications," in [Proc. SPIE: Smart Biomedical and Physiological Sensor Technologies VII], (04 2010).

[6] DeBiasio, M., Arnold, T., and Leitner, R., "Uav-based multi-spectral imaging system for environmental monitoring," Technisches Messen 11(78), 503-507 (2011). 